\title{
Rational Gauss-Chebyshev Quadratures for Wireless Performance Analysis
}

\author{
Yamuna Dhungana and Chintha Tellambura, Fellow, IEEE
}

\begin{abstract}
The convergence rate of the classical GaussChebyshev quadrature (GCQ) rule for wireless performance as a function of the signal-to-noise ratio (SNR) is analyzed. The convergence rate is found to decline as the SNR varies from high to low. Thus, at low SNR, the number of nodes needed to achieve the desired accuracy is extremely high. A generalized rational GCQ rule is thus adopted. The nodes and weights are then computed from a system of orthogonal rational functions. The rational GCQ is much more accurate than the classical GCQ over the entire SNR range. Especially at low SNR, the accuracy is extremely high.
\end{abstract}

Index Terms-Error probability, Gauss-Chebyshev quadrature, moment generating function, outage probability, signal-tonoise ratio.

\section{INTRODUCTION}

G AUSS quadrature is extremely useful for wireless engineers and researchers. For instance, the GaussChebyshev quadrature (GCQ) rule, which is defined as

$$
\int_{-1}^{1} \frac{f(x)}{\sqrt{1-x^{2}}} d x=\sum_{k=1}^{n} w_{k} f\left(x_{k}\right)+R_{n}
$$

where $w_{k}=\pi / n$ and $x_{k}=\cos ((2 k-1) \pi /(2 n))$, is attractive due to its explicit nodes $x_{k}$ and weights $w_{k}$. Note that the error $R_{n}$ becomes zero if $f(x)$ is a polynomial of degree less than or equal to $2 n-1$. The GCQ rule (1) has been used for the error performance analysis of coded systems [1][3], diversity combining systems with binary modulation [4] and M-ary quadrature amplitude modulation (MQAM) [5]. It has also been used for the outage probability in presence of co-channel interference and fading [6] and has many other potential applications.

Surprisingly, the convergence rate (the value of $n$ needed to achieve a given level of accuracy) has never been investigated in the wireless literature. This rate, in fact, degrades significantly in the low signal-to-noise ratio (SNR) region where the poles of $f(x)$ approach the interval $[-1,1]$, and the GCQ rule may require hundreds of nodes for high accuracy. This problem can be solved by using the rational GCQ rule [7]. It has exactly the same form as (1), except for how the nodes $x_{k}$ and the weights $w_{k}$ are computed; a system of orthogonal rational functions is thus utilized instead of the orthogonal polynomials used in the classical GCQ (which, however, yield closed-form $x_{k}$ and $w_{k}$ ). However, the computation is fast and efficient. The rational GCQ rule is exact in a maximal space of rational functions with arbitrary real poles outside $[1,-1]$ [7].

Manuscript received November 15, 2012. The associate editor coordinating the review of this letter and approving it for publication was G. Colavolpe.

The authors are with the Department of Electrical and Computer Engineering, University of Alberta, Edmonton, AB T6G 2V4, Canada (e-mail: yamuna.dhungana@gmail.com).

Digital Object Identifier 10.1109/WCL.2013.012513.120837
For the first time, this letter proposes the use of the rational GCQ rule for wireless performance analysis. Specifically, the contributions of this letter are as outlined below.

1) The convergence of the classical GCQ rule for wireless performance is studied. The impact of SNR on the convergence rate is analyzed and quantified.

2) The rational version of the GCQ rule is shown to be more accurate than the classical one for a given value of $n$ in the entire SNR range. Tremendous reduction in the number of functional evaluations required is achieved in the low SNR region.

3) A simple algorithm (from [7]) to compute the nodes and weights of rational GCQ rule is adopted.

\section{GCQ RULE AND ITS CONVERGENCE}

The GCQ rule for error performance analysis has been derived for specific applications in the past and thus, is available in various forms. We thus highlight how it is used to compute the average $\mathbb{E}[Q(\sqrt{\kappa \gamma})]$, where $Q(x)$ is the Gaussian $\mathrm{Q}$ function, and $\kappa$ is a parameter dependent on the binary modulation scheme. Similar expressions for other modulation schemes are also possible [8]. Given the moment generating function (MGF) of $\gamma, M_{\gamma}(s)=\mathbb{E}[\exp (-s \gamma)]$, the average is

$$
\mathbb{E}[Q(\sqrt{\kappa \gamma})]=\frac{1}{2 \pi} \int_{-1}^{1} \frac{M_{\gamma}(\kappa /(x+1))}{\sqrt{1-x^{2}}} d x .
$$

The expression is derived by applying Craig's formula $Q(\sqrt{\kappa \gamma})=1 / \pi \int_{0}^{\pi / 2} \exp \left(-\kappa \gamma /\left(2 \cos ^{2} \theta\right)\right) d \theta$ followed by the change of variables, $\cos 2 \theta=x$. The expectation can be computed by applying the $n$-node GCQ rule (1), where $f(x)=1 /(2 \pi) M_{\gamma}(\kappa /(x+1))$.

Of fundamental importance, the convergence behavior of the GCQ rule (1) for (2) is now studied. We consider the $N_{r} \geq 1$-branch maximal ratio combining (MRC) system over independent and identically distributed (iid) Rayleigh fading. The MGF of the output SNR $\gamma$ in this case is given by $M_{\gamma}(s)=(1+\bar{\gamma} s)^{-N_{r}}$, where $\bar{\gamma}$ is the average SNR. Thus, the GCQ rule for the average bit error rate (BER) of the binary phase shift keying (BPSK) modulation $(\kappa=2)$ can be readily obtained. The number of nodes required in (1) for a relative error of $10^{-14}$, denoted by $n_{\text {req }}$, is plotted in Figure 1 against $\bar{\gamma}$ for $N_{r}=1$. Clearly, the convergence rate decreases significantly as the SNR $\bar{\gamma}$ decreases, and this rate becomes very slow at low SNR values. To explain this behavior, we bound the error term $R_{n}$ with the help of the following theorem.

Theorem 1. If $f$ is analytic in a domain containing $[-1,1]$ in its interior, the remainder $R_{n}$ in (1) is given by

$$
R_{n}=\frac{1}{2 \pi i} \oint_{\Gamma} f(z)\left(\phi(z)-r_{n}(z)\right) d z,
$$




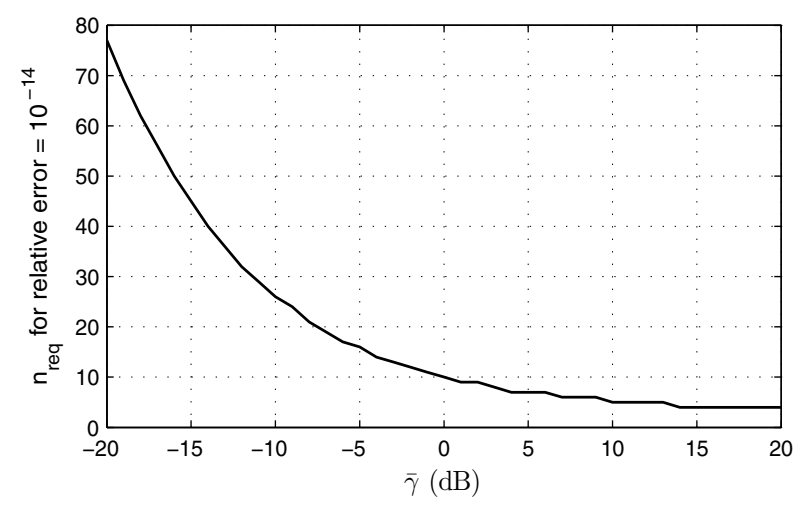

Fig. 1. Impact of average SNR $\bar{\gamma}$ on the convergence rate of GCQ.

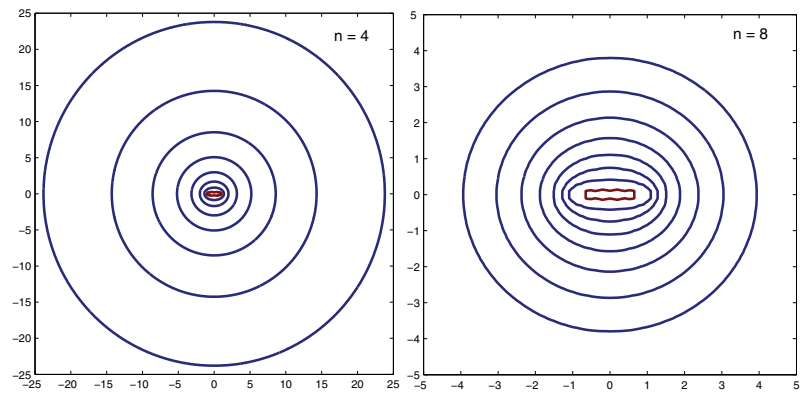

Fig. 2. Contour plots of $\left|\phi(z)-r_{n}(z)\right|$ with $n=4$ and 8 .

where $\Gamma$ is a contour contained in the domain of analyticity of $f$ that encloses $[-1,1]$ once in the counter-clockwise direction, and $\phi(z), r_{n}(z)$ are defined by

$$
\begin{aligned}
\phi(z) & =\int_{-1}^{1} \frac{d x}{\sqrt{1-x^{2}}(z-x)}=-\frac{\pi z \sqrt{\frac{1}{z^{2}}}}{\sqrt{1-z^{2}}}, \\
r_{n}(z) & =\frac{\pi}{n} \sum_{k=1}^{n} \frac{1}{z-\cos \left(\frac{(2 k-1)}{2 n} \pi\right)} .
\end{aligned}
$$

Proof: Apply Cauchy's formula to $f$ in both the integral and the quadrature sum in (1) [9, Theorem 2.48].

From (3), it follows that

$$
\left|R_{n}\right| \leq \frac{l(\Gamma)}{2 \pi} \max _{z \in \Gamma}\left|\phi(z)-r_{n}(z)\right| \cdot \max _{z \in \Gamma}|f(z)|,
$$

where $l(\Gamma)$ is the length of contour $\Gamma$.

The contour plots of $\left|\phi(z)-r_{n}(z)\right|$ with $n=4$ and 8 are shown in Figure 2. The contours correspond to the levels $1,10^{-2}, 10^{-4}, \ldots, 10^{-14}$ from inner to outer. Thus, $\left|\phi(z)-r_{n}(z)\right|$ decreases as the contours move away from $[-1,1]$. Then, for a given value of $n,(6)$ gives a small bound on $R_{n}$ for a contour far from $[-1,1]$. However, the choice of the contour is limited to where $f$ is analytic (i.e., to where there are no poles). Thus, if $f$ is analytic only in a small neighborhood of $[-1,1]$, the bound on $R_{n}$ will not be small due to the closer contour chosen, which indicates that the desired accuracy can be achieved only by increasing $n$. We will now use the example of Figure 1 to explain this theory.

The function is $f(x)=\frac{1}{2 \pi} \frac{(x+1)^{N_{r}}}{(x+1+2 \bar{\gamma})^{N_{r}}}$ with $N_{r}=1$, which has a pole at $-(1+2 \bar{\gamma})$. Thus, the radius $r$ of the circular contour $\Gamma$ chosen should be $1<r<(1+2 \bar{\gamma})$ such that $f$ is analytic in $|z| \leq r$. For $\bar{\gamma}=-10 \mathrm{~dB}$, the region of analyticity is $|z|<1.2$. Figure 3 shows the error bound plots for different

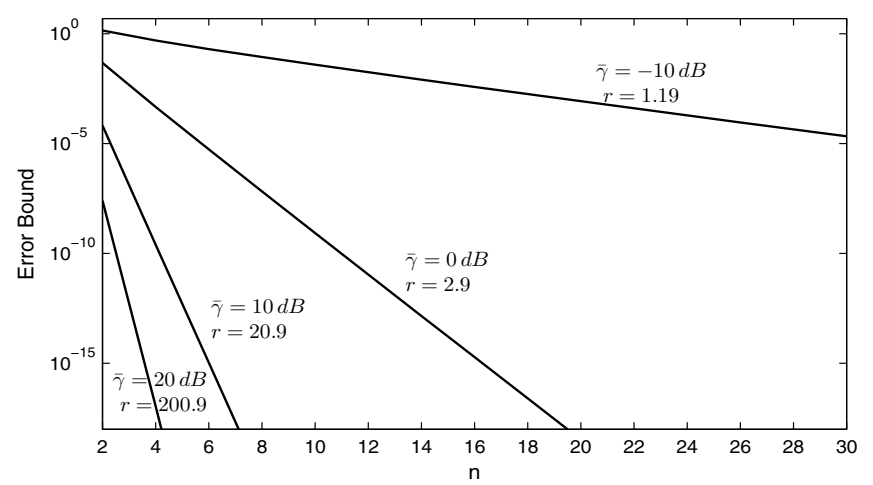

Fig. 3. GCQ error bound for the BER of BPSK over Rayleigh fading

values of $\bar{\gamma}$ by taking the maximum possible value of $r$. The bound derived in [10],

$$
\left|R_{n}\right| \leq \frac{1.05}{r^{2 n-2}(2 n)^{1 / 2}}\left(\frac{1}{r^{4}-1}\right)^{1 / 2}\|f\|
$$

is used for the plot where $\|f\|^{2}=\int_{0}^{2 \pi}\left|f\left(r e^{i \phi}\right)\right|^{2} d \phi$. Figure 3 shows that $\bar{\gamma}$ determines the contour and thus the bound, indicating the slow convergence of GCQ at low SNR values.

Thus, the classical GCQ rule has poor convergence in the low SNR region since the poles of $f(x)$ approach $[-1,1]$. For such integrands, it is more appropriate to consider the GCQ rule based on orthogonal rational functions, rather than orthogonal polynomials, with preassigned poles to simulate the singularities of the integrand [11], [12] (and references therein). Some important results on the rational GCQ rule for the real poles outside $[-1,1]$ are presented in the following section.

\section{RATIONAL GCQ RULE}

Definition 1. Let $\left\{\alpha_{1}, \alpha_{2}, \ldots\right\}$ be a sequence of real numbers outside $[-1,1]$. A set of rational functions of the form

$\varphi_{k}(x)=\frac{b_{k} x^{k}+b_{k-1} x^{k-1}+\ldots+b_{0} x^{0}}{\left(1-x / \alpha_{1}\right)\left(1-x / \alpha_{2}\right) \ldots\left(1-x / \alpha_{k}\right)}, k=1,2, \ldots$

that are all mutually orthogonal with respect to the weight function $W(x)=1 / \sqrt{1-x^{2}}$ on $[-1,1]$ are called Chebyshev orthogonal rational functions on $[-1,1]$ with real poles. If $\alpha_{j}=\infty$ for every $j \geq 1$, then $\varphi_{k}(x)$ in (8) reduces to Chebyshev polynomials of degree $k$.

By using a conformal mapping known as the Joukowski transform $x=1 / 2 \cdot(z+1 / z)$ denoted by $x=J(z)$, which maps the unit circle to the interval $[-1,1]$, explicit expressions for the Chebyshev orthonormal rational functions are derived in [7]. The following theorem is from [7].

Theorem 2. Chebyshev orthonormal rational function on $[-1,1]$ with real poles $\left\{\alpha_{1}, \alpha_{2}, \ldots\right\}$ outside this interval is given by

$\varphi_{n}(x)=\frac{1}{\sqrt{2 \pi}} \sqrt{1-\beta_{n}^{2}}\left(\frac{z B_{n-1}(z)}{1-\beta_{n} z}+\frac{1}{\left(z-\beta_{n}\right) B_{n-1}(z)}\right)$

where $x=J(z), \beta_{k}=J^{-1}\left(\alpha_{k}\right)$ such that $\left\{\beta_{1}, \beta_{2}, \ldots\right\} \subset$ $[-1,1]$, and $B_{n}(z)$ is a Blaschke product given by

$$
B_{n}(z)=\prod_{k=1}^{n} \frac{z-\beta_{k}}{1-\beta_{k} z}, \quad n=1,2, \ldots, \quad B_{0}(z)=1 .
$$


The nodes $\left\{x_{k}\right\}_{k=1}^{n}$ in the $n$-node rational GCQ rule (1) are the zeros of $\varphi_{n}(x)$ and can be computed as $x_{k}=\cos \left(\theta_{k}\right)$ where $\theta_{k}$ is the only zero on $(0, \pi)$ of a real-valued function $g_{n}\left(\theta_{k}\right)$ that strictly increases over the interval $(0, \pi)$ :

$$
\begin{aligned}
g_{n}\left(\theta_{k}\right)= & 2 \sum_{j=1}^{n-1} \arg \cos \theta_{k}-\beta_{j}+i \sin \theta_{k}-(n-1) \theta_{k}+ \\
& \arg \cos \theta_{k}-\beta_{n}+i \sin \theta_{k}-\pi / 2(2 k-1) .
\end{aligned}
$$

The corresponding weights $w_{k}$ in (1) are given by

$$
w_{k}=2 \pi\left(1+h\left(x_{k}\right)\right)^{-1}, \quad k=1,2, \ldots, n,
$$

where

$$
h(x)=2 \sum_{j=1}^{n-1} \frac{\sqrt{1-1 / \alpha_{j}^{2}}}{1-x / \alpha_{j}}+\frac{\sqrt{1-1 / \alpha_{n}^{2}}}{1-x / \alpha_{n}} .
$$

\section{Remarks:}

1) The function $g_{n}\left(\theta_{k}\right)$ is concave on $(0, \pi)$ if the poles are all positive and convex if they are all negative. If there are both positive and negative poles, $g_{n}\left(\theta_{k}\right)$ has only one inflection point on $(0, \pi)$.

2) Since $g_{n}\left(\theta_{k}\right)$ for $k=1,2, \ldots, n$ are negative vertical shifts of a strictly increasing base function $f_{n}\left(\theta_{k}\right)$ by $c_{k}=\pi / 2 \cdot(2 k-1)$ such that $c_{1}<c_{2}<\ldots<c_{n}$, we have $0 \leq \theta_{1}<\theta_{2}<\ldots<\theta_{n} \leq \pi$.

Newton's method can be used for finding the zero $\theta_{k}$ of $g_{n}\left(\theta_{k}\right)$. For our problems of interest, the poles are all negative, and thus, $g_{n}\left(\theta_{k}\right)$ is convex on $(0, \pi)$. For a strictly increasing convex function, the iterations of Newton's method successively decrease towards the root if the initial guess is greater than the actual root. To exploit such a convergence, we find the zeros from $\theta_{n}$ to $\theta_{1}$ with the initial guess $\theta_{n}=\theta_{n+1}=\pi$ and the initial guesses for $\theta_{n-1}, \ldots, \theta_{1}$ set according to the linear extrapolation $\theta_{k-1}=\theta_{k}+\left(\theta_{k}-\theta_{k+1}\right)$.

\section{Applications of Rational GCQ RUle}

\section{A. Error performance of MRC in independent fading}

MRC is a well-known optimal diversity combining method in the absence of interfering signals [8, Chap. 9].

For a MRC receiver in independent fading, the MGF of the output SNR $\gamma$ is given by $M_{\gamma}(s)=\prod_{l=1}^{N_{r}} M_{\gamma_{l}}(s)$, where $M_{\gamma_{l}}(s)$ is the MGF of the $l$-th branch SNR $\gamma_{l}$. From eqn. (2), the average probability of error for the MRC system can be computed by using the rational GCQ rule (1), where $f(x)=$ $1 /(2 \pi) \prod_{l=1}^{N_{r}} M_{\gamma_{l}}(\kappa /(x+1))$. The function $f(x)$ for common fading models is given in Table I. The following examples illustrate the construction and use of the rational GCQ rule for BER analysis of BPSK MRC in different fading channels.

Example 1 (Independent Rayleigh fading): Let $N_{r}=2$ with $\overline{\gamma_{l}}=l \cdot \bar{\gamma}(l=1,2)$. Thus, $f(x)$ has 2 simple poles. The rational GCQ rule can be applied by taking $\alpha_{1}=-(1+2 \bar{\gamma})$, $\alpha_{2}=-(1+4 \bar{\gamma})$ and $\alpha_{k}=\infty$ for $k>2$.

Example 2 (Independent Nakagami- $m$ fading): Let $N_{r}=2$ with $\overline{\gamma_{l}}=l \cdot \bar{\gamma}(l=1,2)$ and $m_{1}=0.8, m_{2}=3.2$. Thus, $f(x)$ has singularities at $-(1+2.5 \bar{\gamma})$ and $-(1+1.25 \bar{\gamma})$ of the fractional orders 0.8 and 3.2 , respectively. These fractional orders are rounded to the nearest integers, and the rational GCQ rule is accordingly applied by taking $\alpha_{1}=-(1+2.5 \bar{\gamma})$, $\alpha_{2}=\alpha_{3}=\alpha_{4}=-(1+1.25 \bar{\gamma})$ and $\alpha_{k}=\infty$ for $k>4$.

\begin{tabular}{|c|c|}
\hline Fading & $f(x)$ \\
\hline \multirow{2}{*}{ Rayleigh } & $1 \quad(x+1)^{N_{r}}$ \\
\hline & $\frac{1}{2 \pi} \frac{1}{\prod_{l-1}^{N_{r}}\left(x+1+\kappa \bar{\gamma}_{l}\right)}$ \\
\hline \multirow{2}{*}{$\begin{array}{l}\text { Nakagami- } \\
m\end{array}$} & \multirow{2}{*}{$\frac{1}{2 \pi} \prod_{l=1}^{N_{r}} \frac{(x+1)^{m_{l}}}{\left(x+1+\kappa \frac{\bar{\gamma}_{l}}{m_{l}}\right)^{m_{l}}}$} \\
\hline & \\
\hline \multirow{2}{*}{ Rician } & $1 \quad(x+1)^{N_{r}} \quad I^{N_{r}} \quad K_{l} \kappa \frac{\gamma_{l}}{K_{l}+1}$ \\
\hline & $\overline{2 \pi} \overline{\prod_{l=1}^{N_{r}}\left(x+1+\kappa \frac{\bar{\gamma}_{l}}{K_{l}+1}\right)} \exp \left(\sum_{l=1} \overline{x+1+\kappa \frac{\bar{\gamma}_{l}}{K_{l}+1}}\right.$ \\
\hline \multirow{2}{*}{ Hoyt } & $1 \frac{(x+1)^{N_{r}}}{1}$ \\
\hline & $+\kappa\left(\frac{2 \overline{\gamma_{l}} q_{l}^{2}}{q_{l}^{2}+1}\right)\left(x+1+\kappa \frac{2 \overline{\gamma_{l}}}{q_{l}^{2}+1}\right)$ \\
\hline
\end{tabular}

TABLE I

THE FUNCTION $f(x)$ FOR $N_{r}$ BRANCH MRC RECEIVER IN SOME COMMON FADING MODELS

TABLE II

RELATIVE ERRORS: $N_{r}=2$, MRC, RAYLEIGH, $\overline{\gamma_{l}}=l \cdot \bar{\gamma}(l=1,2)$

\begin{tabular}{|c|ccc|}
\hline $\bar{\gamma}$ & $\mathrm{n}$ & Rational GCQ & Classical GCQ \\
\hline \multirow{3}{*}{$10 \mathrm{~dB}$} & 2 & $1.0435 \mathrm{e}-015$ & $3.1750 \mathrm{e}-004$ \\
& 4 & $7.0435 \mathrm{e}-015$ & $1.1785 \mathrm{e}-010$ \\
& 8 & $7.3043 \mathrm{e}-015$ & $7.9565 \mathrm{e}-015$ \\
\hline & 2 & $1.1445 \mathrm{e}-016$ & 0.0066 \\
& 4 & $8.0117 \mathrm{e}-016$ & 0.0052 \\
$-10 \mathrm{~dB}$ & 8 & $1.1445 \mathrm{e}-016$ & $5.5695 \mathrm{e}-005$ \\
& 16 & $2.1746 \mathrm{e}-015$ & $2.7853 \mathrm{e}-009$ \\
& 32 & 0 & $5.7226 \mathrm{e}-016$ \\
\hline
\end{tabular}

Example 3 (Rician fading): For a single branch Rician fading channel with $K=3, f(x)$ has a pole of infinite multiplicity at $-(1+0.5 \bar{\gamma})$. We thus take $\alpha_{1}=\alpha_{2}=\ldots=$ $\alpha_{n}=-(1+0.5 \bar{\gamma})$.

Example 4 (Hoyt fading): For a single branch Hoyt fading with $q=0.5, f(x)$ has a branch cut on $[-(1+3.2 \bar{\gamma}),-(1+$ $0.8 \bar{\gamma})$ ]. Thus, $f(x)$ can be approximated by a rational function with poles on the cut. Although there can be many choices for the poles, we consider two distinct poles such that $\alpha_{2 k+1}=$ $\lambda_{1}(k=0,1,2, \ldots)$ and $\alpha_{2 k}=\lambda_{2}(k=1,2, \ldots)$, with $\lambda_{1}$ and $\lambda_{2}$ taken to be the zeros of the second-degree Chebyshev polynomial of the first kind $T_{2}(x)$ transformed to the interval $[-(1+3.2 \bar{\gamma}),-(1+0.8 \bar{\gamma})]$; i.e., $\lambda_{1}=-(1+2 \bar{\gamma})+1.2 \bar{\gamma} / \sqrt{2}$ and $\lambda_{2}=-(1+2 \bar{\gamma})-1.2 \bar{\gamma} / \sqrt{2}$.

The relative errors of the rational GCQ and classical GCQ for these examples are given in Tables II-V, which clearly verify the higher accuracy of the rational GCQ as compared to that of the classical GCQ. Note that each of these examples addresses a unique function (a function with simple poles or multiple poles or essential singularities or branch cut) and thus, illustrates the construction and use of the rational GCQ rule that are applicable to other areas of error performance analysis. Many other potential applications are omitted for brevity.

\section{B. Outage Probability due to co-channel interference}

The outage probability in interference is defined as the probability that the instantaneous signal-to-interference ratio (SIR) falls below a pre-defined threshold $\gamma_{t h}$. Let the instantaneous powers of the desired signal and $N_{I}$ interfering signals be $p_{0}$ and $p_{l}, l=1,2, \ldots, N_{I}$, respectively, with average power $\Omega_{l}$, $l=0,1,2, \ldots, N_{I}$. The SIR is defined as $\Gamma=p_{0} / \sum_{l=1}^{N_{I}} p_{l}$. The outage probability, $P_{\text {out }}=\mathbb{P}\left(\Gamma<\gamma_{t h}\right)=\mathbb{P}(\gamma<0)$, where $\gamma=p_{0} / \gamma_{t h}-\sum_{l=1}^{N_{I}} p_{l}$, can then be expressed as 
TABLE III

RELATIVE ERRORS: $N_{r}=2$, MRC, NAKAGAMI- $m$ FADING, $\overline{\gamma_{l}}=l \cdot \bar{\gamma}(l=1,2)$ AND $m_{1}=0.8, m_{2}=3.2$

\begin{tabular}{|c|ccc|}
\hline $\bar{\gamma}$ & $\mathrm{n}$ & Rational GCQ & Classical GCQ \\
\hline $10 \mathrm{~dB}$ & 4 & $4.1808 \mathrm{e}-011$ & $1.0411 \mathrm{e}-006$ \\
& 5 & $9.8233 \mathrm{e}-015$ & $3.1420 \mathrm{e}-009$ \\
\hline $0 \mathrm{~dB}$ & 4 & $2.6660 \mathrm{e}-008$ & $1.3925 \mathrm{e}-004$ \\
& 10 & $1.6598 \mathrm{e}-016$ & $3.6045 \mathrm{e}-010$ \\
\hline & 4 & $1.2776 \mathrm{e}-007$ & 0.0051 \\
$-10 \mathrm{~dB}$ & 10 & $1.4427 \mathrm{e}-011$ & $9.9404 \mathrm{e}-006$ \\
& 15 & $3.5752 \mathrm{e}-014$ & $2.0009 \mathrm{e}-006$ \\
& 20 & $5.9987 \mathrm{e}-016$ & $5.0039 \mathrm{e}-008$ \\
\hline
\end{tabular}

TABLE IV

RELATIVE ERRORS: $N_{r}=1$, RICIAN FADING $(K=3)$

\begin{tabular}{|c|ccc|}
\hline $\bar{\gamma}$ & $\mathrm{n}$ & Rational GCQ & Classical GCQ \\
\hline $10 \mathrm{~dB}$ & 4 & $3.1224 \mathrm{e}-009$ & $3.3039 \mathrm{e}-009$ \\
& 6 & $1.2536 \mathrm{e}-015$ & $8.7331 \mathrm{e}-013$ \\
\hline \multirow{3}{*}{$0 \mathrm{~dB}$} & 4 & $1.7566 \mathrm{e}-006$ & $8.5131 \mathrm{e}-005$ \\
& 6 & $2.6548 \mathrm{e}-011$ & $4.8141 \mathrm{e}-006$ \\
& 8 & $4.3798 \mathrm{e}-015$ & $7.0533 \mathrm{e}-009$ \\
\hline & 4 & $2.3436 \mathrm{e}-006$ & 0.0045 \\
$-10 \mathrm{~dB}$ & 8 & $2.2259 \mathrm{e}-013$ & $5.4763 \mathrm{e}-004$ \\
& 10 & $1.6567 \mathrm{e}-014$ & $3.5435 \mathrm{e}-005$ \\
\hline
\end{tabular}

$P_{\text {out }}=\frac{1}{2 \pi} \int_{-1}^{1} \frac{\Re\left\{\left(1-j \frac{\sqrt{1-x^{2}}}{1+x}\right) M_{\gamma}\left(c+j c \frac{\sqrt{1-x^{2}}}{1+x}\right)\right\}}{\sqrt{1-x^{2}}} d x$.

The expression is obtained by using [6, Eqn. 8] with the change in variables, $\omega=c \sqrt{1-x^{2}} /(1+x)$, where $0<c<$ $s_{m}, s_{m}$ is the smallest pole of $M_{\gamma}(s)$ in the right-half plane.

Example 5 (Rician fading with multiple Rayleigh interferers) The desired signal experiences Rician fading while $N_{I}$ interfering signals are Rayleigh faded. The MGF of $\gamma$ is

$$
M_{\gamma}(s)=\frac{\exp \left(-\frac{K s \frac{\Omega_{0}}{(K+1) \gamma_{t h}}}{1+\frac{\Omega_{0}}{(K+1) \gamma_{t h}} s}\right)}{\left(1+\frac{\Omega_{0}}{(K+1) \gamma_{t h}} s\right) \prod_{l=1}^{N_{I}}\left(1-\Omega_{l} s\right)} .
$$

Now, to compute the outage (14) by using the rational GCQ rule, we first observe that $f(x)$ has a pole of infinite multiplicity at $-\left(1+\frac{2 \Omega_{0}^{2} c^{2} /\left(\gamma_{t h}^{2}(K+1)^{2}\right)}{1+2 \Omega_{0} c /\left(\gamma_{t h}(K+1)\right)}\right)$ and $N_{I}$ simple poles at $-\left(1+\frac{2 \Omega_{l}^{2} c^{2}}{1-2 \Omega_{l} c}\right), l=1,2, \ldots, N_{I}$. Next, the value of $c$ is chosen such that the poles move away from the interval $[-1,1]$ while still satisfying the condition $0<c<s_{m}$. For the given example, $s_{m}=1 / \max \left(\Omega_{l}\right), l=1,2, \ldots, N_{I}$. If we choose $c=s_{m} / 2$, then one of the poles moves to infinity while all other poles become less than -1 , and the rational GCQ rule can readily be applied. Let us define $\Lambda=\Omega_{0} /\left(\gamma_{t h} \sum_{l=1}^{N_{I}} \Omega_{l}\right)$. Table VI gives the relative error of the rational GCQ rule compared with that of the classical GCQ for different values of $\Lambda$ when $K=2, N_{I}=2, \Omega_{1}=1$ and $\Omega_{2}=1.6$. The efficiency of the classical GCQ degrades as the value of $\Lambda$ decreases. For $\Lambda=-10 \mathrm{~dB}, 300$ nodes are required to achieve a relative error of $10^{-16}$. With the rational GCQ, that level of accuracy can be achieved with only 9 nodes.

\section{CONCLUSION}

We showed that the popular classical GCQ rule for wireless performance converges slowly at low SNRs because the poles of $f(x)$ approach the line segment $[-1,1]$ in the complex plane as the SNR declines. We thus proposed the adoption of the rational GCQ rule. Its use requires the on-the-fly
TABLE V

RELATIVE ERRORS: $N_{r}=1$, HOYT FADING ( $\left.q=0.5\right)$

\begin{tabular}{|c|ccc|}
\hline $\bar{\gamma}$ & $\mathrm{n}$ & Rational GCQ & Classical GCQ \\
\hline $10 \mathrm{~dB}$ & 2 & $2.1708 \mathrm{e}-006$ & $6.2410 \mathrm{e}-005$ \\
& 5 & $7.4169 \mathrm{e}-016$ & $1.0612 \mathrm{e}-012$ \\
\hline & 2 & $1.1478 \mathrm{e}-004$ & 0.0085 \\
$0 \mathrm{~dB}$ & 5 & $2.3639 \mathrm{e}-011$ & $3.4883 \mathrm{e}-006$ \\
& 9 & $3.5177 \mathrm{e}-016$ & $1.7759 \mathrm{e}-010$ \\
\hline & 2 & $1.6553 \mathrm{e}-004$ & 0.0675 \\
$-10 \mathrm{~dB}$ & 5 & $3.4027 \mathrm{e}-010$ & 0.0033 \\
& 7 & $1.5042 \mathrm{e}-013$ & $5.2158 \mathrm{e}-004$ \\
& 10 & $3.9254 \mathrm{e}-015$ & $3.7345 \mathrm{e}-005$ \\
\hline
\end{tabular}

TABLE VI

RELATIVE ERRORS: $K=2, N_{I}=2, \Omega_{1}=1$ AND $\Omega_{2}=1.6$

\begin{tabular}{|c|ccc|}
\hline$\Lambda$ & $\mathrm{n}$ & Rational GCQ & Classical GCQ \\
\hline \multirow{3}{*}{$10 \mathrm{~dB}$} & 3 & $1.3942 \mathrm{e}-004$ & $8.0153 \mathrm{e}-005$ \\
& 6 & $6.5155 \mathrm{e}-013$ & $4.6123 \mathrm{e}-006$ \\
& 9 & $1.5286 \mathrm{e}-014$ & $1.2827 \mathrm{e}-008$ \\
\hline \multirow{3}{*}{$0 \mathrm{~dB}$} & 3 & $3.5539 \mathrm{e}-004$ & 0.0054 \\
& 6 & $2.2556 \mathrm{e}-009$ & $2.9299 \mathrm{e}-004$ \\
& 9 & $1.5424 \mathrm{e}-015$ & $2.7710 \mathrm{e}-005$ \\
\hline \multirow{3}{*}{$10 \mathrm{~dB}$} & 3 & $4.5677 \mathrm{e}-004$ & 0.0125 \\
& 6 & $3.7059 \mathrm{e}-009$ & 0.0054 \\
& 9 & $2.2774 \mathrm{e}-016$ & 0.0029 \\
\hline
\end{tabular}

computation of the nodes and weights, which is fast and efficient with MATLAB. The accuracy of the rational GCQ rule is extremely high; a relative accuracy of $10^{-15}$ is possible for all SNRs and the fading models considered. As this level of accuracy is more than enough for engineering purposes, the rational GCQ rule may even be considered as a replacement for closed-form solutions. Due to space limitations, many potential applications were not explored. However, a vast array of wireless performance problems appear amenable to the rational GCQ rule.

\section{REFERENCES}

[1] E. Biglieri, G. Caire, G. Taricco, and J. Ventura-Traveset, "Simple method for evaluating error probabilities," IEE Elect. Lett., vol. 32, no. 3, pp. 191-192, Feb. 1996.

[2] C. Tellambura, "Evaluation of the exact union bound for trellis-coded modulations over fading channels," IEEE Trans. Commun., vol. 44, no. 12, pp. 1693-1699, Dec. 1996.

[3] G. Taricco and E. Biglieri, "Exact pairwise error probability of spacetime codes," IEEE Trans. Inf. Theory, vol. 48, no. 2, pp. 510-513, Feb. 2002.

[4] A. Annamalai, C. Tellambura, and V. K. Bhargava, "Efficient computation of MRC diversity performance in Nakagami fading channel with arbitrary parameters," IEE Elect. Lett., vol. 34, no. 12, pp. 1189-1190.

[5] A. Annamalai, C. Tellambura, and V. Bhargava, "Exact evaluation of maximal-ratio and equal-gain diversity receivers for M-ary QAM on nakagami fading channels," IEEE Trans. Commun., vol. 47, no. 9, pp. 1335-1344, Sep. 1999.

[6] C. Tellambura and A. Annamalai, "An unified numerical approach for computing the outage probability for mobile radio systems," IEEE Commun. Lett., vol. 3, no. 4, pp. 97-99, Apr. 1999.

[7] J. V. Deun, A. Butheel, and P. G. Vera, "On computing rational gausschebyshev quadrature formulas," Mathematics of Computation, vol. 75, no. 253, pp. 307-326, Oct. 2005.

[8] M. K. Simon and M.-S. Alouini, Digital Communication over Fading Channels, 1st edition. Wiley, 2000.

[9] W. Gautschi, Orthogonal Polynomials Compuatation and Approximation. Oxford University Press Inc., 2004.

[10] F. Stetter, "Error bounds for gauss-chebyshev quadrature," Mathematics of Computation, vol. 22, no. 103, pp. 657-659, July 1968.

[11] J. V. Deun and A. Butheel, "Orthogonal rational functions and quadratures on an interval," J. Computational and Applied Mathematics, vol. 153, no. 1-2, pp. 487-495, Apr. 2003.

[12] K. Deckers and A. Butheel, "The existence and construction of rational gauss-type quadrature rules," Applied Mathematics and Computation, vol. 218, no. 20, pp. 10 299-10 320, June 2012. 